\title{
Fundamental Chemistry and Thermodynamics of Hydrothermal Oxidation Processes
}

Oak Ridge National Laboratory

September 1997

\section{Progress Report}

\section{Principal Investigator}

J. M. Simonson

(423) 574-4962 (Phone)

(423) 574-4961 (Pax)

simonsonjm@ornl.gov

Oak Ridge National Laboratory

P.O. Box 2008, Bldg. 4500S, MS-6110

Oak Ridge, TN 37831-6110

\section{Co-Investigators}

R. E. Mesmer

(423) 574-4958 (Phone)

(423) 574-4961 (Fax)

rem@ornl.gov

Oak Ridge National Laboratory

P.O. Box 2008, Bldg. 4500S, MS-6110

Oak Ridge, TN 3783 1-6110

J. G. Blencoe

(423) 574-7041 (Phone)

(423) 574-4961 (Fax)

coe@oml.gov

Oak Ridge National Laboratory

P.O. Box 2008, Bldg. 4500S, MS-6110

Oak Ridge, TN 37831-6110
P. T. Cummings

(423) 241-4779 (Phone)

(423) 574-6843 (Fax)

u53@ornl.gov

Oak Ridge National Laboratory

P.O. Box 2008, Bldg. 4500S, MS-6110

Oak Ridge, TN 37831-6110
A. A. Chialvo
(423) 974-5242 (Phone)
(423) 974-4910 (Fax)
achialvo@utk.edu
University of Tennessee
Knoxville, TN 37966

\section{Contributors}

S. Dai, ORNL

J. C. Seitz, ORNL 


\section{Research Objective}

The objective of this research program is to provide fundamental scientific information on the physical and chemical properties of solutes in aqueous solutions at high temperatures needed to assess and improve the applicability of hydrothermal oxidation (HTO) to the remediation of U.S. Department of Energy (DOE) hazardous and mixed wastes. Investigators in two divisions at Oak Ridge National Laboratory (Chemical and Analytical Sciences, and Chemical Technology) and at the University of Tennessee are focused on the solubility and speciation of actinides and surrogates in model HTO process streams at high temperatures, on the experimental and theoretical development of equations of state for aqueous mixtures containing noncondensible gases under HTO process conditions ranging above the critical temperature of water, and on achieving a predictive level of understanding of the chemical and physical properties of HTO process streams through molecular-level simulations of aqueous solutions at high temperatures.

Specific tasks in these three efforts over the past year include measurements of solubility and identification of stable solid phases for $\mathrm{UO}_{3}$ in aqueous carbonate solutions at temperatures above $100^{\circ} \mathrm{C}$, measurements of fluid-phase coexistence boundaries and densities of mixtures in $\left\{\mathrm{H}_{2} \mathrm{O}+\mathrm{N}_{2}+\mathrm{CO}_{2}\right\}$ mixtures at high temperatures and pressures, and molecular dynamics simulations of water and aqueous solutions addressing the speciation of "simple" ionic solutes and the structure of water and aqueous solutions as functions of temperature and density.

\section{Research Statement}

Research in this project has been divided into individual tasks, each addressing a particular scientific question and each contributing to a unified understanding of HTO processing problems related to the treatment of DOE hazardous and mixed wastes. The three primary tasks are (1) the determination of solubilities of inorganic compounds including actinides and surrogates to determine their likely fate during HTO processing, (2) experimental and modeling studies of the density and phase behavior of $\{$ water + gas $\}$ mixtures at high temperatures to determine the' physical state of the process fluid, and (3) simulations of water and aqueous solutions at high temperatures and comparison with experimental results as a method for the development of accurate, comprehensive descriptions of the properties of aqueous fluids.

- Inorganic compounds (e.g., salts and oxides) become insoluble in aqueous solutions at sufficiently high temperatures, but quantitative knowledge of solubility phenomena is lacking for actinides at elevated temperatures. This information is central to a determination of the suitability of HTO processing for various DOE wastes and for process design and modeling considerations as the technology is developed for application to these wastes. Solubility studies of actinide oxides [U(VI)] in aqueous solutions at high temperature were begun, with emphasis on identification of stable solid phases at high temperature, to address one area where new experimental results are needed.

- Noncondensible gases (e.g., $\mathrm{N}_{2}, \mathrm{O}_{2}$ and $\mathrm{CO}_{2}$ ) will be present as products or reactants from HTO processing of DOE wastes, and the density and phase behavior of these gases in aqueous mixtures affect directly important operating parameters including heat and mass transfer, single and multiphase flow regions, and fluid retention time in the reaction zone. 
Existing data for these systems are neither extensive nor precise enough for reliable interpolation or for development of equations of state, particularly near the critical point of water $\left(374^{\circ} \mathrm{C}, 221 \mathrm{bars}\right)$. Precise determinations of density and phase-coexistence boundaries for binary and ternary aqueous systems were carried out during the reporting period at temperatures and pressures near the critical point of water.

- Neither equations of state nor standard treatments for thermodynamic properties of aqueous electrolyte solutions can be extrapolated reliably beyond the range of available data, and purely theoretical treatments of aqueous systems have not resulted in a predictive capability. Simulations from which both structural and energetic properties of aqueous systems can be calculated based on the microscopic behavior of model systems with appropriately chosen intermolecular and intramolecular potential functions hold great promise for the accurate description of complex mixtures over wide ranges of state variables (temperature, density, and composition). Advances are being made in the description of speciation (i.e., ion-pair formation) in aqueous solutions at high temperature through the comparison of simulation studies with experimental results.

Additional details of the progress made during FY 1997 on these tasks are given in the following sections.

\section{Research Progress}

\section{Solubility Measurements}

Methods were developed to measure conveniently for the first time solubilities of uranium oxide $\left(\mathrm{UO}_{3}\right)$ in aqueous solutions at elevated temperatures and to determine the identity of the solid uranium-containing phase in equilibrium with the fluid. Noting that $\mathrm{CO}$, produced from oxidation of organic waste compounds will yield carbonates and bicarbonates in solution if base is added to neutralize acids produced during the process, initial experiments focused on solubilities in (carbonate + bicarbonate) buffered solutions with and without additional $\mathrm{NaCl}$.

Method Development and Application. The conventional (quench) method for solubility determinations as applied to these studies involves heating a sealed silica tube containing $\mathrm{UO}_{3}(\mathrm{~s})$ and carbonate buffer solution in a constant-temperature aluminum block, followed by quenching to ambient temperature. After quenching, the tube is opened immediately and filtered to separate the sample solution from excess solid. The concentration of uranyl ion in the solution is then determined spectrophotometrically. Equilibrium can be approached either from a supersaturated solution via precipitation or from an undersaturated solution via dissolution, and agreement of results from both determinations increases confidence that equilibrium has been established. Analysis of results at $155^{\circ} \mathrm{C}$ indicated that the undersaturated samples approached equilibrium within about 1 week, while 2-3 weeks were needed for the supersaturated samples [with $\mathrm{UO}_{3}(\mathrm{cr}$ ) added to provide nucleation sites] to reach equilibrium. The apparent reversibility of the solubility reaction indicated that reliable results could be obtained from undersaturated solutions, resulting in relatively shorter equilibriation times.

A second method involving continuous monitoring of uranyl species in solution at high temperature by ultraviolet-visible spectrophotometry has also been developed in this program. 
The key to this method lies in the use of commercial micro-scale silica flow cells (from Hellma International) that can be thermostated directly in the beam of the spectrophotometer. This setup is expected to permit measurements on aqueous solutions up to $400^{\circ} \mathrm{C}$. The present assumption that the optical molar absorption coefficient is independent of temperature will be checked through experiments on standardized solutions as functions of temperature.

Additional in situ measurements will be completed before preparation of a short paper describing the apparatus and techniques used for these studies. The in situ method has significant advantages over the conventional (quench) method in that the sample concentrations can be monitored continuously, assuring that equilibrium has been attained and providing some kinetic information from the equilibration run.

Early analyses by X-ray diffraction of the solid phase recovered from equilibrium experiments indicated that the phases produced at high temperatures were amorphous. This prompted us to choose micro-scale diffuse reflectance Fourier-transform infrared spectroscopy (DR IR) as a primary technique to analyze structures of these solid phases. DR IR spectra were obtained for a number of $\mathrm{U}(\mathrm{VI})$ solid phases including $\mathrm{UO}_{3}, \mathrm{UO}_{2} \mathrm{CO}_{3}$, and $\mathrm{Na}_{4} \mathrm{UO}_{2}\left(\mathrm{CO}_{3}\right)_{3}$; these phases are all possible candidates for solids in equilibrium with the test solutions at elevated temperatures. These spectra, along with DR IR spectra of several other uranium oxide species, form a DR IR spectral library of various uranium solid-state phases that will be used to analyze various uranium equilibrium solid phases from future solubility experiments.

Solubility Results. The solubility of $\mathrm{UO}_{3}(\mathrm{~s})$ in $0.1 \mathrm{M}$ carbonate buffer $\left(\mathrm{NaHCO}_{3} / \mathrm{Na}_{2} \mathrm{CO}_{3}\right)$ was measured as a function of temperature and ionic strength (as $\mathrm{NaCl}$ ) from 112 to $149^{\circ} \mathrm{C}$. It was found that the solubility decreased with increasing temperature. At $112^{\circ} \mathrm{C}$, the solubility first increases with increasing $\mathrm{NaCl}$ concentration, then decreases with further increases in ionic strength. However, at the higher temperatures investigated the solubility increased monotonically with ionic strength. DR IR spectra of the solids from high temperature exhibited signals characteristic of $\mathrm{CO}_{3}{ }^{2-}$. This indicates that the solid phase may convert from $\mathrm{UO}_{3}$ to $\mathrm{UO}_{2} \mathrm{CO}_{3}$ or $\mathrm{Na}_{4} \mathrm{UO}_{2}\left(\mathrm{CO}_{3}\right)_{3}$ because of the reaction of $\mathrm{UO}_{3}(\mathrm{~s})$ with $\mathrm{Na}_{2} \mathrm{CO}_{3}$. The low solubility of $\mathrm{UO}$, in the buffer solution resulted from the decrease of carbonate concentration because of the above solid/liquid reaction. Future experiments will be carried out with a higher liquid/solid mass ratio to minimize variation of the $\mathrm{pH}$ and total carbonate concentration in the liquid phase.

Optical spectroscopic measurement of the dissolution process of UO, into the buffer solution revealed that the uranyl concentration in the buffer solution at $145^{\circ} \mathrm{C}$ increases rapidly in the first day, followed by a steady decrease to less than $10^{-3} \mathrm{~mol} \cdot \mathrm{kg}^{-1}$. This is consistent with our model of the dissolution reaction, where the initial driving force for the dissolution of $\mathrm{UO}_{3}$ comes from the complexation of $\mathrm{UO}_{2}{ }^{2+}$ with $\mathrm{CO}_{3}{ }^{2-}$. Gradually, the carbonate ion in the solution is consumed by the reaction between solid $\mathrm{UO}_{3}$ and carbonate ion, thereby reducing the solubility of $\mathrm{UO}_{3}$ in the buffer solution. Solubilities of $\mathrm{Na}_{4} \mathrm{UO}_{2}\left(\mathrm{CO}_{3}\right)_{3}$ (cr) in the buffer solution are currently under investigation to address the possible identity of the stable solid phase of $\mathrm{U}(\mathrm{VI})$ at high temperature without the complication of changing carbonate activity in the solution.

\section{Fluid-Phase PVT Properties and Fluid+FIuid Phase Equilibria}

Using a unique, custom-designed, high-pressure, vibrating-tube densimeter, we are performing experiments to determine the densities, excess molar volumes and fluid+fluid (solvus) relations of $\mathrm{O}_{2}-\mathrm{N}_{2}-\mathrm{CO}_{2}-\mathrm{H}_{2} \mathrm{O}$ fluids at $250-500^{\circ} \mathrm{C}$ and $50-1000$ bars. This year we acquired numerous data for $\mathrm{N}_{2}-\mathrm{CO}_{2}-\mathrm{H}_{2} \mathrm{O}$ fluids at $300^{\circ} \mathrm{C}$ and pressures from 75 to 1000 bars. 
Densities and Excess Volumes. At $300^{\circ} \mathrm{C}$ and pressures from 75 to 500 bars, the densities of $\mathrm{N}_{2}-\mathrm{CO}_{2}-\mathrm{H}_{2} \mathrm{O}$ fluids increase from approximately 0.037 to $0.777 \mathrm{~g} \cdot \mathrm{cm}^{-3}$. Densities of the ternary fluid mixtures are not accurately predicted from data for the unary and binary fluids. Excess molar volumes, calculated from our new density data, are continuously positive, of intermediate magnitude (less than $30 \mathrm{~cm}^{3} \cdot \mathrm{mol}^{-1}$ ), and slightly asymmetric toward $\mathrm{H}_{2} \mathrm{O}$ at pressures immediately below the saturation vapor pressure of water ( $\left.\mathrm{p}_{\text {sat }} \approx 85 \mathrm{bars}\right)$; highly asymmetric toward $\mathrm{H}_{2} \mathrm{O}$ and very large (greater than $280 \mathrm{~cm}^{3} \cdot \mathrm{mol}^{-1}$ ) at pressures immediately above $\mathrm{p}_{\text {sat }}$; and strongly asymmetric toward the $\mathrm{CO}_{2}-\mathrm{N}_{2}$ binary, but small (less than $\pm 4 \mathrm{~cm}^{3} \cdot \mathrm{mol}^{-1}$ ), at 1000 bars. This complex behavior indicates large and highly pressure-dependent deviations from ideal mixing.

Solvus Relations. Solvus datawere obtained at numerous pressures between $\mathrm{p}_{\text {sat }}$ and 1000 bars. Results indicate that the range of fluid-fluid immiscibility is strongly dependent on the $\mathrm{N}_{2} / \mathrm{CO}_{2}$ ratio, extending from $\mathrm{p}_{\text {sat }}$ to pressures above 1000 bars in the $\mathrm{N}_{2}-\mathrm{H}_{2} \mathrm{O}$ system, but from $\mathrm{p}_{\text {sat }}$ to only -570 bars in the $\mathrm{CO}_{2}-\mathrm{H}_{2} \mathrm{O}$ system, where $\mathrm{CO}$; becomes completely miscible with water at $300^{\circ} \mathrm{C}$. In the $\mathrm{N}_{2}-\mathrm{H}_{2} \mathrm{O}$ system, the field of immiscibility widens steadily with increasing pressure up to 1000 bars. In the $\mathrm{CO}_{2}-\mathrm{H}_{2} \mathrm{O}$ system, the solvus expands rapidly between $\mathrm{p}_{\text {sat }}$ and 300 bars, then narrows steadily with increasing pressure up to the critical point at about 570 bars. Therefore, in the ternary system, the vapor- and liquid-saturation curves extend all the way from the $\mathrm{N}_{2}-\mathrm{H}_{2} \mathrm{O}$ binary to the $\mathrm{CO}_{2}-\mathrm{H}_{2} \mathrm{O}$ sideline for pressures between $\mathrm{p}_{\text {sat }}$ and 570 bars.

In addition, our data suggest that these phase boundaries are nearly linear at pressures immediately above $\mathrm{p}_{\text {sat }}$, but with increasing pressure up to 570 bars, the liquid-saturation curve becomes distinctly curvilinear. From 570 to 1000 bars, the critical point of the $\mathrm{N}_{2}-\mathrm{CO}_{2}-\mathrm{H}_{2} \mathrm{O}$ solvus migrates from the $\mathrm{CO}_{2}-\mathrm{H}_{2} \mathrm{O}$ sideline toward more $\mathrm{N}_{2}$-rich compositions. Its exact location in this pressure range is difficult to determine experimentally because coexisting phases near the critical point have similar densities.

\section{Molecular Dynamics Simulation Studies of Aqueous Systems at High Temperatures}

Simulation of Ion Speciation in Supercritical Water. Molecular simulations of infinitely dilute $\mathrm{NaCl}$ aqueous solutions have been performed to study the $\mathrm{Na}^{+} \mathrm{Cl}$ ion pairing in a polarizable and a nonpolarizable solvent at supercritical conditions. The simple point charge (SPC), the Pettitt-Rossky, and the Fumi-Tosi models for the water-water, the ion-water, and the ion-ion interactions respectively were used in the determination of the degree of dissociation, its temperature and density dependence, and the kinetics of the interconversion between ion-pair configurations in a nonpolarizable medium.

To assess the effect of the solvent polarizability on the stability of the ion-pair configurations, we replaced the SPC model with the polarizable point charge water (PPC) model and determined the anion-cation potential of mean force at reduced temperature (absolute temperature divided by critical temperature) of 1.20 and reduced density (density divided by critical density) of 1.5 .

Surprisingly, better results are obtained using the SPC model than the PPC model, suggesting the need for more accurate intermolecular potential models not only for water but also for the ions in solution.

Solvation in High-temperature Electrolyte Solutions. Our previously developed molecular-based formalism for infinitely dilute supercritical nonelectrolyte solutions was extended to electrolyte solutions at finite concentrations by establishing rigorous connections 
between the microscopic behavior of the solvent around individual ionic species and their macroscopic solvation behavior.

This formalism relies on the unambiguous splitting of the mixture's properties into short(finite) and long-ranged (diverging) contributions, associated with the corresponding solvation and compressibility-driven phenomena, respectively. Consequently, the finiteness of the residual chemical potentials of species in solution is linked to their solvent's local environment, and the solvation contribution to the salt partial-molar properties can now be interpreted in terms of the short-ranged, salt-solvent and solvent-solvent direct correlation function integrals.

\section{Summary of Accomplishments}

- Data on the solubility of potassium carbonate at high temperatures have been analyzed and a paper describing the results has been accepted for publication. These results will be important to the modeling of solid solubilities in an HTO process that incorporates neutralization of acids in the high-temperature fluid.

- Water has profound and complex effects on the density-pressure-composition relations of $\mathrm{N}_{2}$ $\mathrm{CO}_{2}-\mathrm{H}_{2} \mathrm{O}$ mixtures at high subcritical temperatures. Measured excess molar volumes of these fluids at pressures immediately above the saturation vapor pressure of pure water are very large, are strongly dependent on fluid composition, and cannot be estimated reliably from any "simple" equation of state.

- Methods have been developed for the determination of solubilities of solid phases of U(VI) in aqueous solutions at high temperatures. Studies to date in (carbonate + bicarbonate) buffer solutions indicate the importance of an accurate knowledge of carbonate concentration in solution and likely conversion of $\mathrm{UO}_{3}(\mathrm{cr})$ to carbonate-containing stable solid phases at high temperatures.

- The effect of solvent polarizability on the speciation and association kinetics of ions in supercritical water was assessed for the first time. The results suggest further work will be necessary to improve both the ion-ion and ion-solvent interaction models if polarizability is to be successfully taken into account.

- A theory was developed to describe the separation between short-range and long-range solvation effects in supercritical electrolyte solutions. The theory is an extension of the highly successful formalism developed in our group to describe infinitely dilute supercritical electrolyte solutions.

\section{Papers and Abstracts}

Blencoe, J. G., L. M. Anovitz, and J. C. Seitz. "Serious Shortcomings of Semiempirical Equations of State for High-Temperature Aqueous C-O-H-N Fuids." Accepted for publication in Geological Society of America Abstracts with Programs. 
Chialvo, A. A., P. T. Cummings, J. M. Simonson, and R. E. Mesmer. "Thermodynamics and Kinetics of Ion Speciation in Supercritical Aqueous Solutions: A Molecular Based Study." Submitted for publication in Fluid Phase Equilibria.

Moore, R. C., R. E. Mesmer, and J. M. Simonson. "The Solubility of Potassium Carbonate in Water Between 384 and 529 K Measured Using the Synthetic Method." Journal of Chemical Engineering Data. In press.

Seitz, J. C. and J. G. Blencoe. "Experimentally Determined Volumetric Properties and Solvus Relations for $\mathrm{H}_{2} \mathrm{O}-\mathrm{CO}_{2}-\mathrm{N}_{2}$ Mixtures at $300^{\circ} \mathrm{C}$ and Pressures $<1000$ bars." Accepted for publication in Geological Society of America Abstracts with Programs. 